\title{
NONLINEAR ANTI-COMMUTING MAPS OF STRICTLY TRIANGULAR MATRIX LIE ALGEBRAS
}

\author{
ZHENGXIN CHEN
}

Abstract. Let $N(\mathbb{F})$ be the Lie algebra consisting of all strictly upper triangular $(n+1) \times(n+$ 1) matrices over a field $\mathbb{F}$. A map $\varphi$ on $N(\mathbb{F})$ is called to be anti-commuting if $[\varphi(x), y]=$ $-[x, \varphi(y)]$ for any $x, y \in N(\mathbb{F})$. We show that for $n \geqslant 4$, a nonlinear map $\varphi: N(\mathbb{F}) \rightarrow N(\mathbb{F})$ is anti-commuting if and only if there exist $b, b_{1}, b_{2} \in \mathbb{F}$ and a nonlinear function $f: N(\mathbb{F}) \rightarrow \mathbb{F}$ such that $\varphi=a d\left(b E_{2 n}\right)+\mu_{b_{2}}^{(n, n+1)}+\mu_{b_{1}}^{(12)}+\varphi_{f}$, where $a d\left(b E_{2 n}\right)$ is an inner anti-commuting map, $\mu_{b_{2}}^{(n, n+1)}, \mu_{b_{1}}^{(12)}$ are extremal anti-commuting maps, $\varphi_{f}$ is a central anti-commuting map.

Mathematics subject classification (2010): 15A04, 15A27, 15A86.

Keywords and phrases: Strictly upper triangular matrices, anti-commuting maps.

\section{REFERENCES}

[1] D. Benkovič, Biderivations of triangular algebras, Lin. Alge. Appl. 431 (2009): 1587-1602.

[2] D. BENKOVIČ AND D. EREMITA, Commuting traces and commutativity preserving maps on triangular algebras, J. Algebra 280 (2004): 797-824.

[3] J. Bounds, Commuting maps over the ring of strictly upper triangular matrices, Lin. Alge. Appl. 507 (2016): 132-136.

[4] M. BREŠAR, Commuting maps: a survey, Taiwanese J. Math. 8 (2004): 361-397.

[5] M. BREŠ AR, On generalized biderivations and related maps, J. Algebra 172 (1995): 764-786.

[6] M. BREŠAR, Commuting traces of biadditive maps, commutativity-preserving mappings and Lie mappings, Trans. Amer. Math. Soc. 335 (1993): 525-546.

[7] M. BREŠ AR AND C. R. Miers, Commuting maps on Lie ideals, Comm. Algebra 23 (1995): 5539_ 5553.

[8] M. BREŠAR AND P. ŠEMRL, Commuting traces of biadditive maps revisited, Comm. Algebra 31 (2003): 381-388.

[9] L. CHEN AND J. ZHANG, Nonlinear Lie derivations on upper triangular matrices, Linear Multilinear Alge. 56 (2008): 725-730.

[10] Z. CHEN, Biderivations and linear commuting maps on simple generalized Witt algebras over a field, Electronic J. Linear Algebra 31 (2016): 1-12.

[11] Z. CHEN AND D. WANG, Nonlinear maps satisfying derivability on standard parabolic subalgebras of finite-dimensional simple Lie algebras, Linear Multilinear Alge. 59 (2011): 261-270.

[12] Z. CHEN AND B. WANG, Commuting automorphisms and derivations of certain nilpotent Lie algebras over commutative rings, Comm. Algebra 43 (2015): 2044-2061.

[13] W. Cheung, Commuting maps of triangular algebras, J. London Math. Soc. 63 (2001): 117-127.

[14] N. Divins KY, On commuting automorphisms of rings, Trans. Roy. Soc. Canada. Sect. III. 49 (1995): 19-22.

[15] G. DolinaR, Maps on upper triangular matrices preserving Lie products, Linear Multilinear Alge. 55 (2007): 191-198.

[16] P. LeE, T. Wong, J. Lin And R. WAng, Commuting traces of multiadditive mappings, J. Algebra 193 (1997): 709-723.

[17] T. LEE AND T. LEE, Commuting additive mappings in semiprime rings, Bull. Inst. Math. Acad. Sinca 24 (1996): 259-268. 
[18] J. LUH, A note on commuting automorphisms of rings, Amer. Math. Monthly 77 (1970): 61-62.

[19] L. MOLNÁR AND P. ŠEMRL, Non-linear commutativity preserving maps on self-adjont operators, Q. J. Math. 56 (2005): 589-595.

[20] P. ŠEmRL, Nonlinear commutativity preserving maps, Acta. Sci. Math. (Szeged) 71 (2005): 781-819.

[21] X. QI AND J. Hou, Nonlinear strong commutativity preserving maps on prime rings, Comm. Algebra 38 (2010): 2790-2796.

[22] X. QI AND J. Hou, Nonlinear strong commutativity preserving maps on triangular algebras, Operators Matrices 1 (2012): 147-158.

[23] J. Vukman, Commuting and centralizing mappings in prime rings, Proc. Amer. Math. Soc. 109 (1990): 47-52.

[24] D. WANG AND X. YU, Biderivations and linear commuting maps on the Schrödinger-Virasoro Lie algebra, Comm. Algebra 41 (2013): 2166-2173.

[25] D. WANG, X. YU AND Z. CHEN, Biderivations of the parabolic subalgebras of simple Lie algebras, Comm. Algebra 39 (2011): 4097-4104.

[26] D. WANG, Y. ZHAO AND Z. ChEN, Non-linear maps on simple Lie algebras preserving Lie products, Comm. Algebra 39 (2011): 424-434.

[27] D. WANG, W. ZHANG AND Z. Chen, Product zero derivations of the parabolic subalgebras of simple Lie algebras, J. Lie Theory 20 (2010): 167-174.

[28] J. Zhang, S. Feng, H. Li And R. Wu, Generalized biderivation of nest algebras, Lin. Alge. Appl. 418 (2006): 225-233. 